\title{
Crónica
}

\section{ACTA DE LA COMISIÓN DE PREMIOS: AÑO 2008}

En Santiago de Chile, con fecha 3 de Noviembre de 2009, a las 08:00 horas, se reúne la Comisión de Premios, formada de acuerdo a los artículos 26, 27 y 29 de los Estatutos de la Sociedad Chilena de Obstetricia y Ginecología.

El Jurado constituido por el Presidente de la Sociedad Dr. Eugenio Suárez P., por los Miembros de la Comisión Dr. Pablo Escudero B. y Dr. Enrique Guiloff F., y por el Secretario General, Dr. Hernán Muñoz S., éste último con derecho a voz solamente; acuerdan otorgar los siguientes premios correspondientes a los trabajos publicados en la Revista Chilena de Obstetricia y Ginecología durante el año 2008:

1.Premio "DR. JORGE DÍAZ BRAVO" año 2008, al mejor trabajo de ingreso: Este premio se declaró desierto.

2. Premio "DR. JUAN MALFANTI AMIGO" año 2008, al mejor trabajo de casuística: "CURVAS DE BIOMETRÍA FETAL CON EDAD GESTACIONAL DETERMINADA POR ECOGRAFÍA DE PRIMER TRIMESTRE. CLÍNICA ALEMANA DE SANTIAGO," de los Drs. Julio Astudillo D., Masami Yamamoto C., Jorge Carrillo T., Max Polanco G., Alfredo Hernández A. Daniel Pedraza S., Paris Valentini Ch., Juan Valdivia M., Álvaro Insunza F., de la Unidad de Medicina Materno fetal, Departamento de Obstetricia y Ginecología, Clínica Alemana de Santiago, Universidad del Desarrollo.

3. Mención Honrosa del Premio "DR. JUAN MALFANTI AMIGO" año 2008 al trabajo: "ESCISIÓN DE LA ZONA DE TRANSFORMACIÓN EN LESIONES DE CÉRVIX: ANÁLISIS DE 1.141 CASOS", de los Drs. Jorge Cabrera D., Juan Villanueva G., Fernando Heredia M., Lionel Correa S., Juan Landeros S., Juanita Navarrete V., Francisco Mucientes H., Rodrigo Klassen P., Clemencia Cabrera F., Margarita Burgos G., Ivonne Riquelme E., Maritza Flores O. Departamento y Servicio de Obstetricia y Ginecología, Departamento de Patología, Departamento de
Salud Pública, Universidad de Concepción, Hospital Guillermo Grant Benavente, Concepción.

4. Premio "DR. JUAN WOOD WALTERS" año 2008, al mejor trabajo de Ginecología: "MANEJO QUIRÚRGICO DEL TERATOMA MADURO: ¿LAPAROSCOPIA O LAPAROTOMÍA?", de los Drs. Paulina Merino O., Francisca de Jourdan H., Pilar Valenzuela M., Roger Gejman E., Cristián Pomés C., Guillermo Durruty V., Mauricio Cuello F. Programa de Obstetricia y Ginecología, Pontificia Universidad Católica de Chile; Programa de Obstetricia y Ginecología, Hospital San Borja Arriarán, Universidad de Chile; Departamento de Obstetricia y Ginecología, Departamento de Anatomía Patológica, Escuela de Medicina, Pontificia Universidad Católica de Chile.

5. Mención Honrosa del Premio "Dr. Juan Wood Walters" año 2008, al trabajo: "NUEVO SISTEMA DE MEDICIÓN URODINAMICA EN LA EVALUACIÓN DE LA INCONTINENCIA URINARIA FEMENINA. EXPERIENCIA PRELIMINAR CON URODINAMIA MONOCANAL CON MEDICIÓN DE PRESIÓN DE RETRO-RESISTENCIA URETRAL", de los Drs. Vicente Solá D., Jack Pardo S., Paolo Ricci A., David Cohen S. Unidad de Uroginecología y Cirugía Vaginal, Departamento de Obstetricia y Ginecología, Clínica Las Condes.

6. Premio "DR. VÍCTOR MANUEL GAZITÚA GUZMÁN" año 2008, al mejor trabajo de Obstetricia: "LONGITUD CERVICAL Y FIBRONECTINA EN EL SINTOMA DE PARTO PREMATURO", de los Drs. Carlos Treuer P., Víctor Quiroz G., Jorge Cabrera D., Carmen Soto L., Heriberto Araneda C. Servicio de Obstetricia y Ginecología, Hospital Guillermo Grant Benavente, Departamento de Obstetricia y Ginecología, Facultad de Medicina, Universidad de Concepción.

7. Premio "DR. CARLOS MONCKEBERG BRAVO" año 2008, al mejor trabajo de Perinatología: "EFECTO DEL MASAJE TERAPÉUTICO EN PRE- 
MATUROS DEL SERVICIO DE NEONATOLOGÍA DEL HOSPITAL SAN JOSÉ", de los Drs. Elsa Rugiero P., Roderick Walton L., Fabiola Prieto P., Eduardo Bravo A., Juan Núñez M., Juan Márquez N., Germán Mühlhausen M. Escuela de Medicina, Facultad de Ciencias Médicas, Universidad de Santiago de Chile, Servicio de Neonatología, Hospital
San José, Facultad de Medicina, Universidad de Chile.

Para dejar constancia firman: DR. ENRIQUE GUILOFF F. DR. PABLO ESCUDERO B. DR. HERNÁN MUÑOZ S. DR. EUGENIO SUÁREZ P. 